\title{
Depositación atmosférica de nitrógeno en un transecto valle longitudinal-cordillera de Los Andes, centro-sur de Chile
}

\author{
Atmospheric deposition of nitrogen in a transect from the Central Valley to Cordillera \\ de Los Andes, south-central Chile
}

CARLOS E. OYARZÚN ${ }^{1}$, ROBERTO GODOY² \& SERGIO LEIVA ${ }^{3}$

Institutos de Geociencias¹, Botánica² y Microbiología3 ${ }^{3}$ Universidad Austral de Chile, Casilla 567, Valdivia, Chile; e-mail ${ }^{1}$ : coyarzun@uach.cl

\begin{abstract}
RESUMEN
El aumento de las actividades agrícolas y ganaderas en el centro-sur de Chile puede producir elevadas emisiones de nitrógeno hacia la atmósfera (mayormente $\mathrm{NH}_{3}$ ). El amoniaco es transportado por corrientes de aire y depositado en el suelo y vegetación. El presente estudio evalúa la depositación atmosférica de compuestos nitrogenados $\left(\mathrm{NO}_{3}{ }^{-} \mathrm{y} \mathrm{NH}_{4}{ }^{+}\right)$ en la química de las precipitaciones en un transecto altitudinal de $66 \mathrm{~km}$, desde los alrededores de la ciudad de Osorno $\left(40^{\circ} 35^{\prime} \mathrm{S}, 72^{\circ} 57^{\prime} \mathrm{O}, 55 \mathrm{~m}\right.$ de altitud) hasta la cordillera de Los Andes, Parque Nacional Puyehue (40 $46^{\circ} \mathrm{S}, 7^{\circ} 11^{\prime}$ O, $1.120 \mathrm{~m}$ de altitud). En siete sitios, durante el período anual junio-1999 hasta mayo-2000, se registró la precipitación y mensualmente se colectaron muestras de agua para determinar: $\mathrm{pH}$, conductividad y las concentraciones de $\mathrm{NO}_{3}^{-} \mathrm{y}$ $\mathrm{NH}_{4}^{+}$. La precipitación anual se incrementó desde $1.103 \mathrm{~mm}$ en la zona de Osorno hasta $6.799 \mathrm{~mm}$ en el sector Antillanca, Parque Nacional Puyehue. Los valores promedio anual del pH registraron escasa variación desde 6,3 en el valle central hasta 5,8 en la cordillera de Los Andes. La conductividad presentó los máximos valores en la proximidad de Osorno $\left(22,9 \mu \mathrm{S} \mathrm{cm}^{-1}\right)$ para descender a través del transecto en zonas intermedias y alcanzar en la Cordillera de los Andes un valor de $11,3 \mu \mathrm{S} \mathrm{cm}^{-1}$. Las concentraciones promedio de $\mathrm{NO}_{3}-\mathrm{N}$ en la lluvia fluctuaron entre $52,3 \mu \mathrm{g} \mathrm{L}^{-1}$ en la zona agrícola-ganadera y $6,9 \mu \mathrm{g} \mathrm{L}^{-1}$ en los bosques del Parque Nacional Puyehue. Las concentraciones de $\mathrm{NH}_{4}-\mathrm{N}$ variaron entre $699,4 \mu \mathrm{g} \mathrm{L}^{-1}$ en el sector de Osorno y $37,8 \mu \mathrm{g} \mathrm{L}^{-1}$ en la cordillera de Los Andes. Los valores de $\mathrm{N}$ inorgánico poseen una marcada tendencia estacional en el sector agrícola, con valores máximos en primavera-verano y los mínimos en invierno. Las tasas anuales de depositación atmosférica de $\mathrm{NO}_{3}-\mathrm{N}$ fluctuaron entre 0,53 y $0,57 \mathrm{~kg}$ ha ${ }_{1}^{1}$ año ${ }^{-1}$ y las de $\mathrm{NH}_{4}-\mathrm{N}$ fueron entre 6,4 y $2,8 \mathrm{~kg} \mathrm{ha}^{-1} \mathrm{año}^{-1}$ para los sitios agrícola-ganaderos de Osorno y Parque Nacional Puyehue, respectivamente. Los resultados se discuten en relación con los posibles efectos ambientales de las crecientes tasas de depositación atmosférica, sobre la acidificación y eutroficación del suelo y aguas subterráneas y de escorrentía, en el valle longitudinal del centro-sur de Chile.
\end{abstract}

Palabras clave: depositación, $\mathrm{NO}_{3}^{-}, \mathrm{NH}_{4}^{+}$, química del agua, agricultura-ganadería, bosque templado lluvioso, Nothofagus.

\section{ABSTRACT}

Agricultural-livestock activities in south-central Chile, could result in elevated emissions of inorganic $\mathrm{N}\left(\mathrm{mainly}_{3} \mathrm{NH}_{3}\right)$ compounds into the atmosphere. Ammonia can be transported via air currents and deposited on the vegetation and soil. The present study evaluates the atmospheric deposition of nitrogen compounds $\left(\mathrm{NO}_{3}^{-} \mathrm{y} \mathrm{NH}_{4}^{+}\right)$in the precipitation, in an altitudinal transect of $66 \mathrm{~km}$ long, from the vicinity of Osorno $\left(40^{\circ} 35^{\prime} \mathrm{S}, 72^{\circ} 57^{\prime} \mathrm{W}, 55 \mathrm{~m}\right.$ of altitude) to Puyehue National Park, Cordillera de Los Andes ( $40^{\circ} 46^{\prime} \mathrm{S}, 7^{\circ}{ }^{\circ} 11^{\prime} \mathrm{W}, 1,120 \mathrm{~m}$ of altitude). In seven sites, from June-1999 to May-2000, precipitation was recorded and water samples were collected monthly, for to determine $\mathrm{pH}$, conductivity and $\mathrm{NO}_{3}{ }^{-}$and $\mathrm{NH}_{4}^{+}$concentrations. Annual precipitation increased from 1,103 mm near Osorno to $6,799 \mathrm{~mm}$ in Antillanca, Puyehue National Park. Annual mean values of $\mathrm{pH}$ showed little change from 6.3 in the central valley to 5.8 in the Cordillera de Los Andes. Conductivity showed the maximum values near Osorno $\left(22.9 \mu \mathrm{S} \mathrm{cm}^{-1}\right)$, intermediate values in transitional sites, and $11.3 \mu \mathrm{S} \mathrm{cm}^{-1}$ in the Cordillera de Los Andes. The annual mean concentrations of $\mathrm{NO}_{3}$ $\mathrm{N}$ changed between $52.3 \mu \mathrm{g} \mathrm{L}^{-1}$ in the agriculture-cattle area and $6.9 \mu \mathrm{g} \mathrm{L}^{-1}$ in temperate rainforests in Puyehue National Park. $\mathrm{NH}_{4}^{+}$concentrations changed between $699.4 \mu \mathrm{g} \mathrm{L}^{-1}$ in the Osorno sector to $37.8 \mu \mathrm{g} \mathrm{L}^{-1}$ in Cordillera de Los Andes. Inorganic- $\mathrm{N}$ values have a marked seasonal variation in the agricultural region with the maximum values in springsummer and minimum in wintertime. Annual rates of atmospheric deposition of $\mathrm{NO}_{3}-\mathrm{N}$ fluctuated between 0.53 and $0.57 \mathrm{~kg} \mathrm{ha}^{-1} \mathrm{yr}^{-1}$, and the $\mathrm{NH}_{4}-\mathrm{N}$ between 6.4 and $2.8 \mathrm{~kg} \mathrm{ha}^{-1} \mathrm{yr}^{-1}$ for the agricultural region of Osorno and Puyehue National Park, respectively. These results are discussed in relation to the potential environmental effects of the increasing atmospheric deposition of nitrogen on the acidification and eutrophication of the soil and groundwater and runoff in the central valley of south-central Chile.

Key words: atmospheric deposition, $\mathrm{NO}_{3}{ }^{-}, \mathrm{NH}_{4}{ }^{+}$, water chemistry, agriculture-cattle, temperate rainforest, Nothofagus. 


\section{INTRODUCCIÓN}

El ingreso de elementos al ecosistema ocurre principalmente a través de la atmósfera vía depositación seca y húmeda o por fijación biológica activa ( $\mathrm{CO}_{2}$ y nitrógeno) contribuyendo en forma importante al ciclo biogeoquímico (Hedin \& Hetherington 1996). En el período pre-industrial el nitrógeno era el factor limitante más importante para el crecimiento en los ecosistemas terrestres no agrícolas y, en especial, los bosques templados a escala mundial, pero en las últimas décadas su aporte vía atmósfera se ha incrementado en el ciclo global, con depositaciones considerables a escala continental (Galloway 1995, Rennenberg \& Gessler 1999).

Con el creciente interés por los problemas ambientales globales, una cantidad considerable de investigación en las últimas décadas ha procurado evaluar y proyectar el impacto de las altas tasas de depositación atmosférica, particularmente de compuestos nitrogenados $\left(\mathrm{NO}_{\mathrm{x}} \mathrm{y} \mathrm{NH}_{\mathrm{y}}\right)$, asociados con actividades antropogénicas, ya que estos elementos son causantes de la acidificación y eutroficación de suelos y cuerpos de aguas con el consecuente desbalance de nutrientes en los ecosistemas (Reynolds et al. 1998, Sutton et al. 1998). El amoniaco atmosférico $\left(\mathrm{NH}_{3}\right)$, junto con el dióxido de azufre $\left(\mathrm{SO}_{2}\right)$ y óxidos de nitrógeno $\left(\mathrm{NO}_{2}\right)$, son los contaminantes primarios que contribuyen a la acidificación de la depositación atmosférica (Sutton et al. 1998).

El N atmosférico de origen antropogénico, está constituido por compuestos oxidados de nitrógeno ( $\mathrm{NO}, \mathrm{NO}_{2} \mathrm{y} \mathrm{NO}_{3}^{-}$), fundamentalmente como producto de la quema de combustibles fósiles, tráfico vehicular y procesos industriales. Los compuestos reducidos de nitrógeno $\left(\mathrm{NH}_{3}\right.$ y $\left.\mathrm{NH}_{4}^{+}\right)$, se producen mayoritariamente en áreas con agricultura y ganadería intensiva (Fangmeier et al. 1994, Rennenberg \& Gessler 1999). El $\mathrm{NH}_{3}$ atmosférico, no sólo puede causar daños a la vegetación próxima a las fuentes de emisión sino que, a mayor escala, contribuye significativamente a la eutroficación por los altos montos producidos y posterior conversión en amonio $\left(\mathrm{NH}_{4}^{+}\right)$, que pueden enriquecer áreas remotas por transporte atmosférico a largas distancias.

La influencia marítima sobre las precipitaciones es de especial importancia en el sur de Chile, ya que los vientos dominantes del oeste transportan las masas de aire provenientes del Océano Pacífico (Hedin et al. 1995, Oyarzún et al. 1998). Antecedentes sobre los constituyentes químicos de las precipitaciones, realizados en el sur de Chile, han señalado que reflejarían una de las aproximaciones más cercanas a condiciones pre- industriales del mundo (Likens et al. 1987, Galloway et al. 1996). Algunos estudios realizados en la cordillera de La Costa de la región centro-sur de Chile ( $\left.40-42^{\circ} \mathrm{S}\right)$, se han referido a la influencia oceánica sobre la química de las precipitaciones (Hedin \& Hetherington 1996), y se han estimado tasas de depositación de nitrógeno inorgánico $\left(\mathrm{NO}_{3}-\mathrm{N}+\mathrm{NH}_{4}-\mathrm{N}\right)$ en áreas boscosas de la cordillera de La Costa $\left(40-42^{\circ} \mathrm{S}\right)$ inferiores a $1 \mathrm{~kg}$ ha $^{-1}$ año $^{-1}$ (Hedin et al. 1995, Oyarzún et al. 1998, Pérez et al. 1998), valores menores a los registrados para zonas con escasa contaminación en Europa (Torseth \& Semb 1997). Estudios recientes sobre flujos de agua y química de las precipitaciones en bosques de Nothofagus pumilio (Poepp. Et Endl.) Krasser, ubicados a $1.120 \mathrm{~m}$ de altitud en la cordillera de Los Andes (40 46' S), indican un ingreso de nitrógeno inorgánico vía precipitación húmeda de $3,3 \mathrm{~kg} \mathrm{ha}^{-1}$ año $^{-1}$ (Godoy et al. 1999). Por último, estudios sobre la química de las precipitaciones en bosques siempreverde de Nothofagus betuloides (Mirb.) Oerst. situados a $920 \mathrm{~m}$ de altitud en la misma localidad, señalan aportes de $3,6 \mathrm{~kg} \mathrm{ha}^{-1} \mathrm{año}^{-1}$, atribuido en parte a la influencia de las actividades de origen antropogénico que se desarrollan en el valle longitudinal de la región centro-sur de Chile (Godoy et al. 2001).

En la actualidad, en el valle longitudinal del centro-sur de Chile se concentran las redes viales, centros urbanos y se desarrolla una dinámica y creciente actividad agropecuaria y forestal, las cuales se están extendiendo hacia ambas laderas cordilleranas. Por su aporte al PGB nacional y regional, los principales rubros son la producción de leche y carne. Antecedentes de Anrique et al. (1999) señalan para la Décima Región de Chile una contribución cercana al $70 \%$ de la producción lechera nacional. Estimaciones de los aportes de nitrógeno para la región, considerando sólo los purines del ganado lechero durante las horas de ordeña es de 2.200 ton año-1, además de los aportes por estabulación durante el período de invierno (J. Dumont comunicación personal). Como producto de estas actividades agrícolas y ganaderas, se producen emisiones de compuestos nitrogenados, que pueden ser depositados en áreas cercanas a las fuentes de emisión (vía depositación seca) o transportados a largas distancias por las corrientes de aire provenientes del Océano Pacífico y depositados sobre la vegetación (vía depositación húmeda). El enriquecimiento en las concentraciones del agua de precipitación, sería significativamente menor en las áreas ubicadas a mayor distancia y altitud en la cordillera de Los Andes. 
El objetivo del presente estudio es evaluar a través de un período anual los cambios en el $\mathrm{pH}$, conductividad y concentraciones de nitrógeno inorgánico $\left(\mathrm{NO}_{3}{ }^{-}+\mathrm{NH}_{4}{ }^{+}\right)$en el agua de precipitación para estimar las tasas de depositación de compuestos nitrogenados a lo largo de un transecto altitudinal de $66 \mathrm{~km}$ de distancia, desde los alrededores de la ciudad de Osorno, área con intensa actividad agrícola y ganadera en el valle longitudinal (55 m s.n.m), hasta áreas boscosas menos perturbadas en el Parque Nacional Puyehue, cordillera de Los Andes (1.120 m de altitud).

\section{MATERIALES Y MÉTODOS}

Área de estudio

El área de estudio se encuentra ubicada en la provincia de Osorno (Fig. 1), caracterizada por un clima templado cálido con estación seca en verano en el valle longitudinal y templado lluvioso de montaña en la cordillera andina. En un transecto altitudinal de $66 \mathrm{~km}$ de distancia, se seleccionaron siete sitios de muestreo: tres corresponden a praderas con actividad agrícolaganadera en el valle longitudinal, un sitio ubicado en una zona de transición pradera-bosque y tres sitios en áreas boscosas del Parque Nacional Puyehue, cordillera de Los Andes. En la Tabla 1 se indica la ubicación geográfica y caracterización de los sitios de estudio.
Precipitación, colecta y análisis químico de muestras de agua

Para determinar los montos mensuales y anual de las precipitaciones en el transecto valle longitudinal-cordillera de Los Andes, se recopiló información climatológica de dos estaciones meteorológicas, correspondientes a Osorno y Aguas Calientes, y adicionalmente se instaló un pluviógrafo en Antillanca (Tabla 1). Para los sitios intermedios, la precipitación fue estimada utilizando interpolación lineal (Schulz 1989).

Para el análisis químico del agua de precipitación, se colectaron muestras compuestas en cada localidad desde 3 pluviómetros (superficie 200 $\mathrm{cm}^{2}$ ). Se instalaron a $1,5 \mathrm{~m}$ de altura sobre el suelo, en lugares ampliamente despejados en los sitios con praderas ganaderas (sin árboles dentro de un diámetro > $50 \mathrm{~m}$ del punto de muestreo) y en claros vegetacionales en los sitios con bosques ( $\sin$ árboles dentro de un diámetro > $20 \mathrm{~m} \mathrm{del}$ punto de muestreo), siguiendo la metodología de Kleemola \& Söderman (1993). Los pluviómetros se diseñaron con un anillo externo metálico para evitar el depósito de fecas de aves y fueron provistos con un filtro de malla plástica y esferas de vidrio, para prevenir la contaminación con hojas e insectos. Los colectores de plástico de $2 \mathrm{~L}$ se pusieron en el interior de tubos opacos para prevenir la influencia de la luz solar la cual puede promover el crecimiento de algas. Previo a la colecta, los envases colectores fueron lavados con $\mathrm{HCl}$ y posteriormente con agua deionizada, para exponerlos por un máximo de 4 días antes de

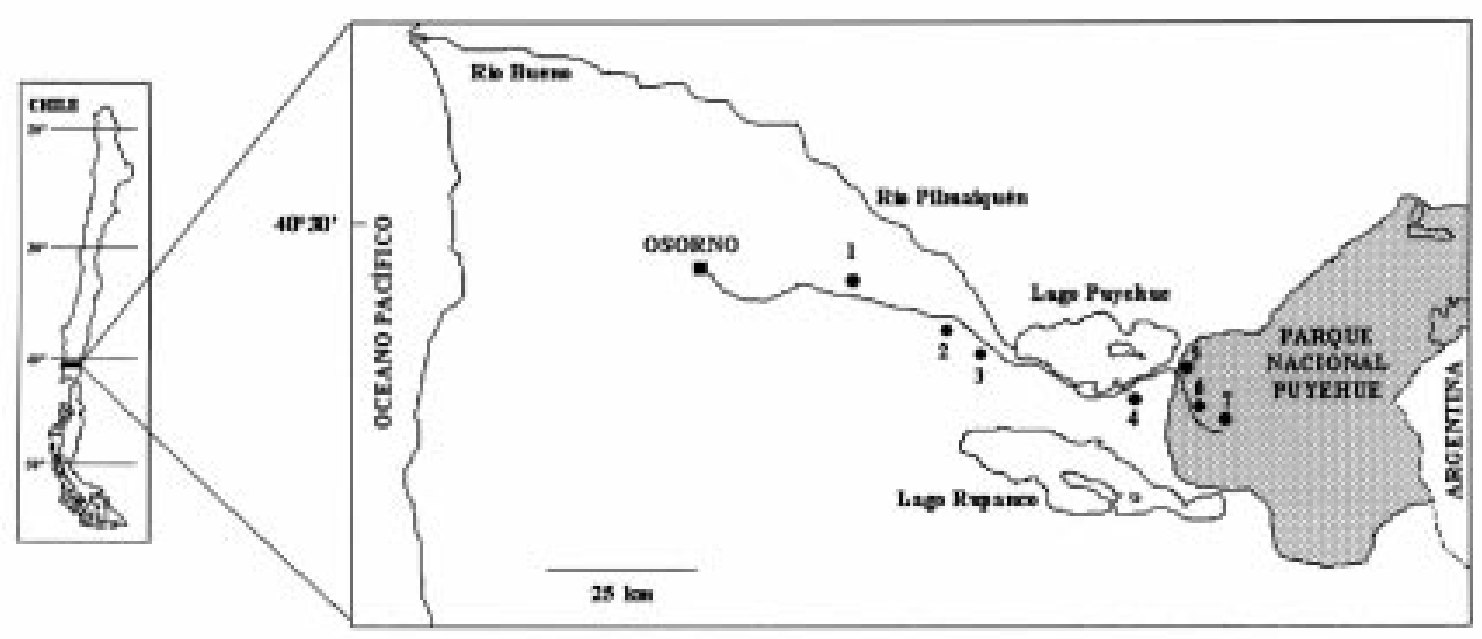

Fig. 1: Ubicación de los sitios de estudio en el transecto valle longitudinal-cordillera de Los Andes, centro-sur de Chile. Los números indican los sitios de muestreo.

Location of the study sites along a transect central valley-Cordillera de Los Andes, south-central Chile. The numbers indicate the sampling sites. 
TABLA 1

Características de los sitios de estudio en el transecto valle longitudinal-cordillera de Los Andes, centro-sur de Chile

Characteristics of the study sites along a transect central valley-Cordillera de Los Andes, south-central Chile

\begin{tabular}{|c|c|c|c|c|c|c|c|}
\hline & Sitio 1 & Sitio 2 & Sitio 3 & Sitio 4 & Sitio 5 & Sitio 6 & Sitio 7 \\
\hline Localidad & Mulpulmo & Tijeral & Entrelagos & La Quema & $\begin{array}{c}\text { Aguas } \\
\text { Calientes }\end{array}$ & Lago Toro & Antillanca \\
\hline $\begin{array}{l}\text { Ubicación } \\
\text { geográfica }(\mathrm{S}, \mathrm{O})\end{array}$ & $\begin{array}{l}40^{\circ} 35^{\prime} 25^{\prime \prime} \\
72^{\circ} 57^{\prime} 07^{\prime \prime}\end{array}$ & $\begin{array}{l}40^{\circ} 36^{\prime} 17^{\prime \prime} \\
72^{\circ} 50^{\prime} 02^{\prime \prime}\end{array}$ & $\begin{array}{l}40^{\circ} 40^{\prime} 58^{\prime}, \\
72^{\circ} 36^{\prime} 33^{\prime}\end{array}$ & $\begin{array}{l}40^{\circ} 41^{\prime} 50^{\prime} \\
72^{\circ} 20^{\prime} 04^{\prime}\end{array}$ & $\begin{array}{l}40^{\circ} 46^{\prime} 30^{\prime \prime} \\
72^{\circ} 15^{\prime} 45^{\prime},\end{array}$ & $\begin{array}{l}40^{\circ} 46^{\prime} 31^{\prime \prime} \\
72^{\circ} 15^{\prime} 45^{\prime},\end{array}$ & $\begin{array}{l}40^{\circ} 46^{\prime} 26^{\prime \prime} \\
72^{\circ} 11^{\prime} 40^{\prime},\end{array}$ \\
\hline Altitud (m) & 55 & 80 & 170 & 195 & 430 & 710 & 1120 \\
\hline Uso del suelo & $\begin{array}{l}\text { Praderas } \\
\text { ganaderas }\end{array}$ & $\begin{array}{l}\text { Praderas } \\
\text { ganaderas }\end{array}$ & $\begin{array}{l}\text { Praderas } \\
\text { ganaderas }\end{array}$ & $\begin{array}{l}\text { Matorral, } \\
\text { transición }\end{array}$ & $\begin{array}{l}\text { Bosque de } \\
\text { Nothofagus }\end{array}$ & $\begin{array}{c}\text { Bosque de } \\
\text { Nothofagus }\end{array}$ & $\begin{array}{l}\text { Bosque de } \\
\text { Nothofagus }\end{array}$ \\
\hline Distancia al mar $(\mathrm{km})$ & 75 & 90 & 100 & 125 & 130 & 135 & 145 \\
\hline Precipitación anual (mm) & 1.103 & 1.635 & 1.779 & 1.930 & 3.130 & 4.393 & 6.799 \\
\hline
\end{tabular}

su recolección. Todas las muestras de lluvia fueron colectadas una vez al mes y transportadas al Laboratorio de Química del Agua, Universidad Austral de Chile en cajas refrigeradas para su análisis antes de $24 \mathrm{~h}$, previa filtración con filtros de $0,4 \mu \mathrm{m}$. En los muestreos no se consideraron eventos de nieve en los sitios de mayor altitud. El $\mathrm{pH}$ y conductividad fueron medidos con electrodos de vidrio (APHA-AWWA-WEF 1995). El análisis de $\mathrm{NO}_{3}^{-}$fue desarrollado mediante el método espectrofotométrico de salicilato de sodio y sal de Seignette. Para el caso del análisis de $\mathrm{NH}_{4}{ }^{+}$se empleó el método de indofenol azul e hipoclorito de sodio (método de Salorzano). Los valores de las concentraciones anuales para cada sitio de estudio se expresan como la media $(\mathrm{n}=$ 12) \pm 1 desviación estándar. Las tasas de depositación de $\mathrm{NO}_{3}{ }^{-}$y $\mathrm{NH}_{4}{ }_{4}^{+}$fueron estimadas combinando los montos de precipitación mensual con la concentración de las formas de nitrógeno para el período correspondiente. Los valores de depositación normalizados se obtuvieron en $\mathrm{g} \mathrm{ha}^{-1} \mathrm{año}^{-1}$ $\mathrm{mm}^{-1}$, con el objetivo de realizar una comparación de los aportes para volúmenes de agua de precipitación equivalente.

Con el objeto de establecer si existen diferencias significativas en las concentraciones de las muestras de agua entre sitios agrícola-ganaderos y bosques andinos durante los períodos de verano e invierno, se utilizó análisis de varianza y la prueba LSD de comparaciones múltiples (Sokal $\&$ Rohl 1981). Las diferencias entre las medias se determinaron a un nivel de $\mathrm{P} \leq 0,05$.

\section{RESULTADOS Y DISCUSIÓN}

La distribución de las precipitaciones anuales a través del transecto para el período junio 1999mayo 2000 muestra un fuerte gradiente desde el valle central hacia la cordillera de Los Andes (Tabla 1). Los sitios 1 al 3 con praderas ganaderas ubicadas en bajas altitudes, registraron una precipitación de 1.103 hasta $1.779 \mathrm{~mm}$ anuales, respectivamente. El sitio 4 (zona de transición), presentó una precipitación anual de $1930 \mathrm{~mm}$, para luego aumentar considerablemente en los sitios 5,6 y 7 con valores de $3.130,4.393$ y 6.799 $\mathrm{mm}$ anual, respectivamente. Mayores antecedentes sobre redistribución de la precipitación en bosques de altura para la región son aportados por Godoy et al. (1999, 2001).

El valor promedio anual de $\mathrm{pH}$ de la lluvia (Fig. 2A), no mostró variaciones significativas entre sitios agrícola-ganaderos y boscosos. Solamente el sitio 1 durante la época de verano, presentó valores significativamente mayores $(\mathrm{P}<0,05)$ que los sitios boscosos. Los valores anuales del pH de la lluvia son similares a los obtenidos por Godoy et al. (2001) en la misma área de estudio, como también para áreas boscosas prístinas de la cordillera de La Costa (Oyarzún et al. 1998). También son semejantes a sitios cercanos al Océano Pacífico del Hemisferio Norte con escasa contaminación y, difieren de los medidos en áreas con fuerte influencia antrópica, donde el $\mathrm{pH}$ es inferior a 5 (Blew \& Edmonds 1995). Con relación a la conductividad, se observa que el mayor valor promedio anual de $22,9 \mu \mathrm{S} \mathrm{cm}^{-1}$ lo presenta el sitio 1 en el valle central, para luego descender a 16,2 , y $13,7 \mu \mathrm{S} \mathrm{cm}^{-1}$ en los sitios 2 y 3 , respec- 
tivamente (Fig. 2B). Desde la zona de transición hacia los bosques de Nothofagus spp. en la cordi1lera de Los Andes, los valores oscilan desde 10,9 hasta $11,4 \mu \mathrm{S} \mathrm{cm}^{-1}$. Estos últimos valores reflejan la menor concentración de iones disueltos en el agua de lluvia y consecuente dilución de los aportes vía precipitación, que provienen desde el Océano Pacífico (Godoy et al. 1999). El análisis estadístico indica que solamente el sitio 1 durante el verano presenta valores de conductividad significativamente mayores $(\mathrm{P}<0,01)$ que los sitios con bosques de la cordillera de Los Andes, ya que durante la época de invierno los valores no difieren significativamente $(\mathrm{P}>0,05)$. El ingreso atmosférico vía depositación seca y húmeda, proveniente de áreas con influencia marina y sin contaminación, poseen por lo general altos valores de conductividad eléctrica y son correlacionados con elevadas concentraciones iónicas, dominadas por sales marinas en que los aerosoles con $\mathrm{Na}^{+}$y $\mathrm{Cl}^{-}$, son los más importantes (Blew \& Edmonds 1995, Hedin \& Hetherington 1996).

Los valores promedio anual de las concentraciones de $\mathrm{NO}_{3}-\mathrm{N}$ registradas en el agua de preci-
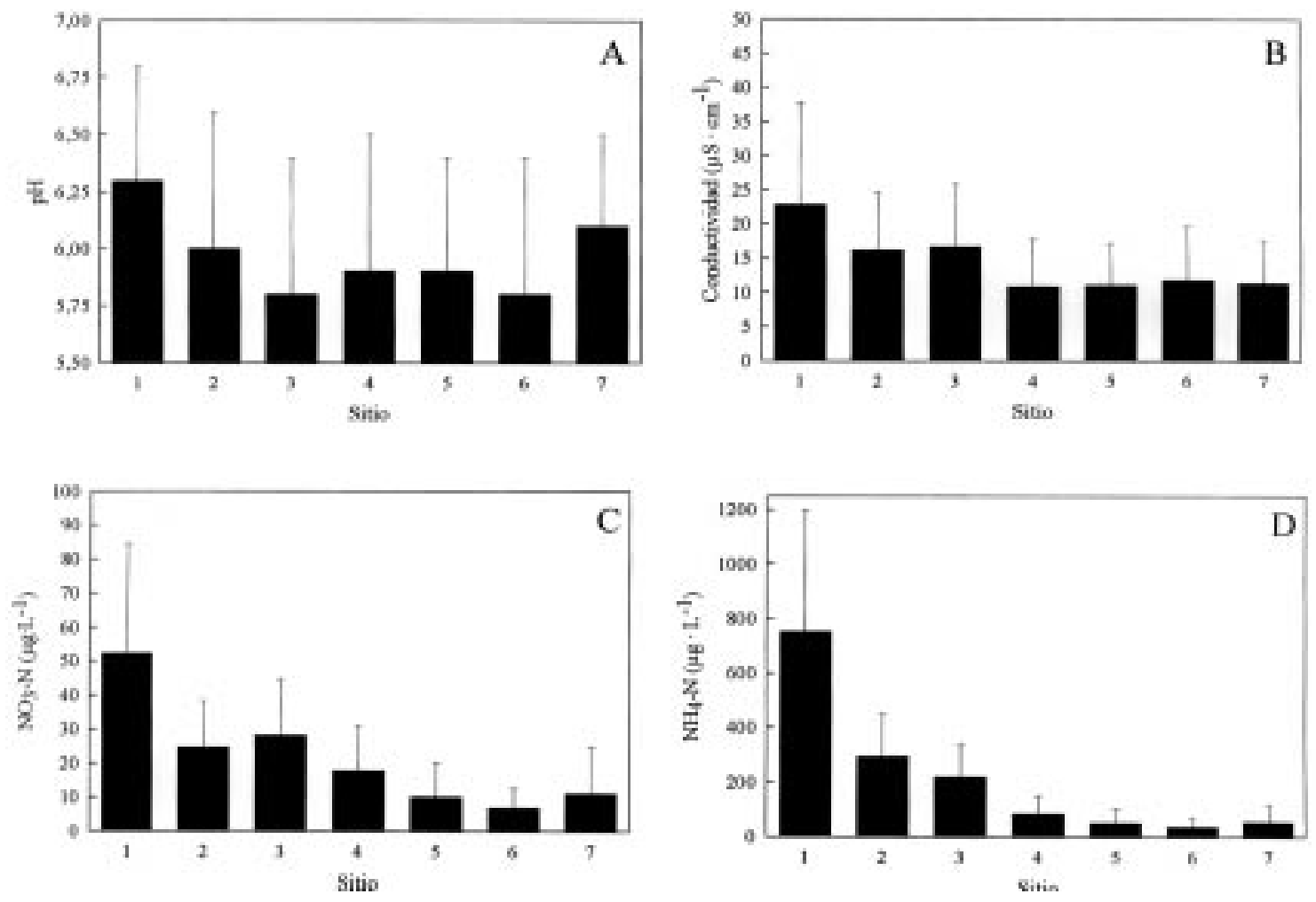

Fig. 2: Valores promedio anual $(\mathrm{n}=12)$ de $\mathrm{pH}(\mathrm{A})$, conductividad $(\mathrm{B})$, nitrato (C) y amonio (D) en el transecto desde sitios agrícola-ganaderos en el valle longitudinal hasta los bosques de la cordillera de Los Andes, centro-sur de Chile.

Annual mean values $(\mathrm{n}=12)$ of $\mathrm{pH}(\mathrm{A})$, conductivity $(\mathrm{B})$, nitrate $(\mathrm{C})$ and ammonium (D) along a transect from agricultural-livestock sites of the central valley to forests in the Cordillera de Los Andes, south-central Chile. 
TABLA 2

Concentraciones de $\mathrm{N}$-inorgánico en el agua de lluvia en zonas boscosas del sur de Chile

Summary of rainfall inorganic-N concentrations in mountainous sites of southern Chile

\begin{tabular}{|c|c|c|c|c|c|c|c|}
\hline Lugar & Período & $\begin{array}{l}\text { Altitud } \\
\quad(\mathrm{m})\end{array}$ & $\begin{array}{l}\text { Precipitación } \\
\left(\mathrm{mm} \text { año }{ }^{-1}\right)\end{array}$ & $\mathrm{pH}$ & $\begin{array}{l}\mathrm{NO}_{3}-\mathrm{N} \\
\left(\mu \mathrm{g} \mathrm{L}^{-1}\right)\end{array}$ & $\begin{array}{l}\mathrm{NH}_{4}-\mathrm{N} \\
\left(\mu \mathrm{g} \mathrm{L}^{-1}\right)\end{array}$ & $\begin{array}{l}\text { Tipo de } \\
\text { bosque }\end{array}$ \\
\hline $\begin{array}{l}\text { 'Parque Nacional } \\
\text { Chiloé }\left(42^{\circ} 30^{\prime} \mathrm{S}\right)\end{array}$ & Enero 1989 & $600-800$ & $\sim 3.000$ & - & $<30,0$ & $<50,0$ & $\begin{array}{l}\text { Fitzroya } \\
\text { cupressoides }\end{array}$ \\
\hline $\begin{array}{l}{ }^{2} \text { Cordillera Pelada } \\
\left(40^{\circ} 05^{\prime} \mathrm{S}\right)\end{array}$ & Abril 1995-marzo 1996 & 820 & 4.098 & 5,4 & 43,2 & - & $\begin{array}{l}\text { Fitzroya } \\
\text { cupressoides }\end{array}$ \\
\hline $\begin{array}{l}{ }^{3} \text { Parque Nacional } \\
\text { Puyehue }\left(40^{\circ} 47^{\prime} \mathrm{S}\right)\end{array}$ & Julio 1997-dic. 1998 & 1.120 & 5.330 & 5,7 & 26,2 & 45,4 & $\begin{array}{l}\text { Nothofagus } \\
\text { pumilio }\end{array}$ \\
\hline $\begin{array}{l}{ }^{4} \text { Parque Nacional } \\
\text { Puyehue }\left(40^{\circ} 47^{\prime} \mathrm{S}\right)\end{array}$ & Abril 1999-marzo 2000 & 1.120 & 6.560 & 6,1 & 9,8 & 39,5 & $\begin{array}{l}\text { Nothofagus } \\
\text { pumilio }\end{array}$ \\
\hline $\begin{array}{l}{ }^{5} \text { Parque Nacional } \\
\text { Torres del Paine } \\
\left(51^{\circ} 10^{\prime} \mathrm{S}\right)\end{array}$ & 1984-1993 & $50-400$ & $\sim 500$ & - & 7,0 & 8,4 & $\begin{array}{c}\text { Nothofagus } \\
\text { pumilio }\end{array}$ \\
\hline $\begin{array}{l}{ }^{6} \text { Lago Rupanco } \\
\left(40^{\circ} 45^{\prime} \mathrm{S}\right)\end{array}$ & Abril 1994-marzo 1995 & $200-1.100$ & 3.325 & - & 27,2 & 52,5 & $\begin{array}{c}\text { Nothofagus } \\
\text { dombeyi }\end{array}$ \\
\hline
\end{tabular}

${ }^{1}$ Hedin \& Campos (1991), ${ }^{2}$ Oyarzún et al. (1998), ${ }^{3}$ Godoy et al. (1999), ${ }^{4}$ Godoy et al. $(2001),{ }^{5}$ Weathers \& Likens

(1997), ${ }^{6}$ Oyarzún et al. (1997)

durante el invierno solamente el sitio 1 mostró diferencias significativas $(\mathrm{P}<0,01)$. En general, los bajos valores de nitrato a través del transecto, reflejan una escasa influencia de las actividades industriales en la zona. En otras áreas del Hemisferio Norte altamente impactadas por actividades antropogénicas, se registran concentraciones de $\mathrm{NO}_{3}^{-}$en el agua de precipitación, que son superiores en varios ordenes de magnitud, a los valores obtenidos en el presente estudio. De Schrijver et al. (2000) en estudios realizados en Bélgica, indican que las concentraciones de nitrato en el agua de precipitación son de hasta $5 \mathrm{mg} \mathrm{L}^{-1}$ y que además son comparativamente más bajas que el agua que llega al piso del bosque (precipitación directa hasta $30 \mathrm{mg} \mathrm{L}^{-1}$ ), hecho atribuido a la contribución vía depositación seca y la eficiente capacidad de intercepción por el dosel de los árboles.

Las concentraciones promedio anual de $\mathrm{NH}_{4}-\mathrm{N}$ en el agua de precipitación presentaron una declinación muy acentuada a través del transecto, con $699,4 \mu \mathrm{g} \mathrm{L}^{-1}$ para el sitio 1 y 295,2 y $220,2 \mu \mathrm{g} \mathrm{L}-$ ${ }^{1}$ para los sitios 2 y 3 , respectivamente, los cuales muestran diferencias altamente significativas ( $P$ $<0,01)$ de los valores de los sitios de áreas boscosas. Si se compara el comportamiento de los datos entre verano e invierno, las diferencias son especialmente significativas durante el verano ( $P$ $<0,001)$ ya que durante el invierno el sitio 3 no difiere significativamente de los sitios con bos- ques $(\mathrm{P}>0,05)$. En la Cordillera de los Andes, los valores descienden desde $84,4 \mu \mathrm{g} \mathrm{L}^{-1}$ en la zona de transición (sitio 4), a 37,8 y $56,2 \mu \mathrm{g} \mathrm{L}^{-1}$, en 1 os sitios 6 y 7, respectivamente (Fig. 2D). Estos valores poseen un comportamiento similar a otras áreas con vegetación boscosa del sur de Chile, encontrándose valores mínimos de referencia en el Parque Nacional Torres del Paine, con 8,4 $\mu \mathrm{g}$ $L^{1}$ (Tabla 2). Galloway et al. (1996) en un estudio sobre la química de las precipitaciones en áreas remotas del Hemisferio Sur, registraron bajas concentraciones de $\mathrm{NH}_{4}+$ y una marcada estacionalidad, con aumentos de los aportes en verano. Sin embargo, los resultados del presente estudio, pueden considerarse relativamente bajos al compararlos con zonas de Europa, de menor precipitación, y con mayor carga ganadera donde la concentración de $\mathrm{NH}_{4}+$ en el agua de precipitación alcanza 1-3 $\mathrm{mg} \mathrm{L}^{-1}$ (Fangmeier et al. 1994).

Las concentraciones de nitrógeno inorgánico en las precipitaciones presentaron una marcada estacionalidad, especialmente en el sitio $1 \mathrm{del}$ Valle Longitudinal, con valores máximos de $\mathrm{NO}_{3}$ $\mathrm{N}$ en el período primavera - verano (octubre $=111$ $\mu \mathrm{g} \mathrm{L}^{-1}$ ) y mínimos en invierno (junio $=20 \mu \mathrm{g} \mathrm{L}^{-1}$ ) (Fig. 3A). Una situación similar se registró con el $\mathrm{NH}_{4}-\mathrm{N}$, con valores máximos en verano (diciembre $\left.=1583 \mu \mathrm{g} \mathrm{L}^{-1}\right)$ y mínimos en invierno (julio $=$ $357 \mu \mathrm{g} \mathrm{L}^{-1}$ ) (Fig. 3B). La variación estacional en la concentración de compuestos nitrogenados reducidos $\left(\mathrm{NH}_{\mathrm{y}}\right)$ ha sido documentada en áreas con- 

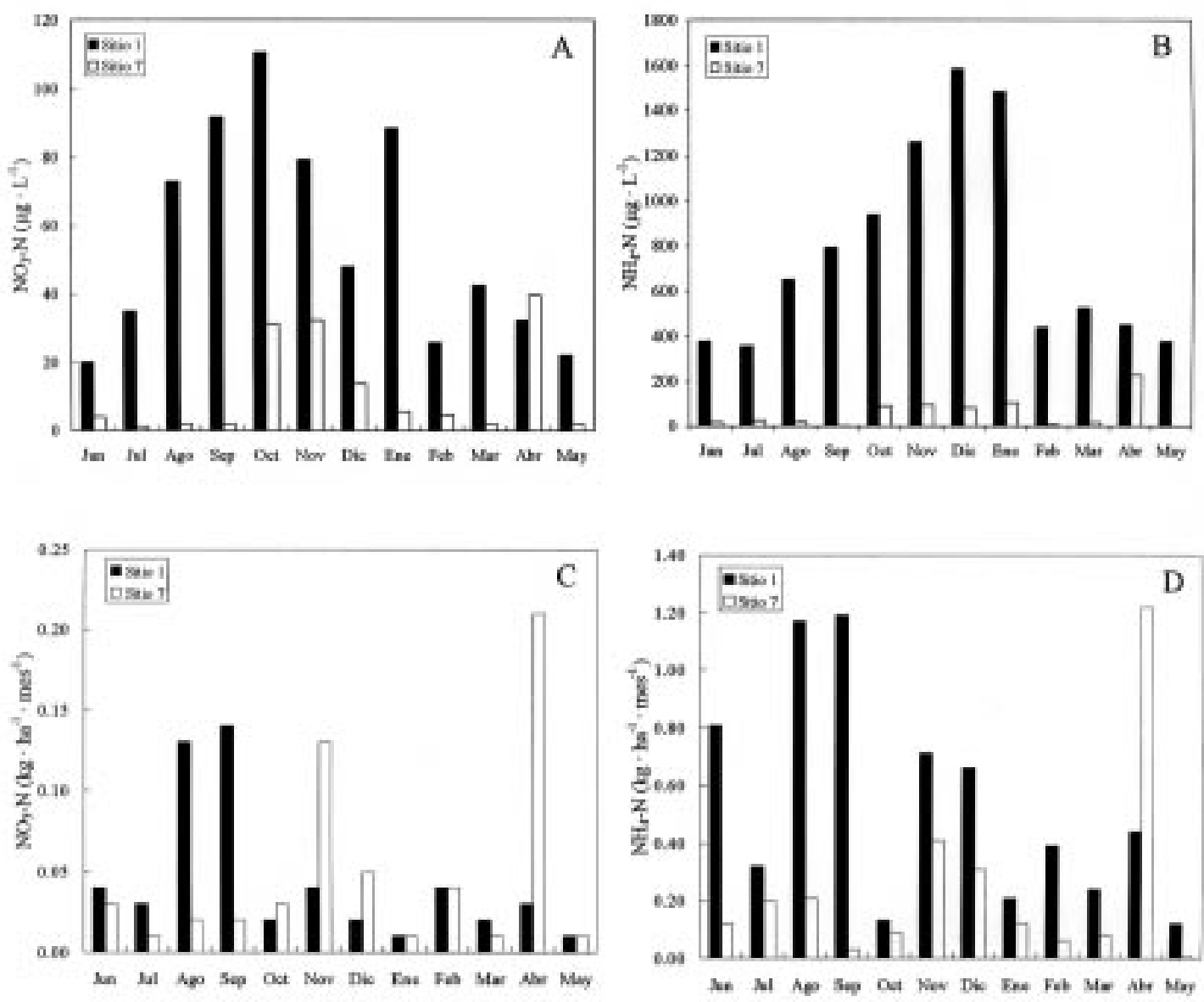

Fig. 3: Comparación de las concentraciones (parte superior) y tasas de depositación (parte inferior) mensuales de nitrógeno inorgánico $\left(\mathrm{NO}_{3}-\mathrm{N}\right.$ y $\left.\mathrm{NH}_{4}-\mathrm{N}\right)$, entre el sitio 1 (valle longitudinal) y el sitio 7 (cordillera de Los Andes), centro-sur de Chile.

Concentrations (above) and monthly depositation rates (below) of inorganic nitrogen $\left(\mathrm{NO}_{3}-\mathrm{N}\right.$ and $\left.\mathrm{NH}_{4}-\mathrm{N}\right)$, between site 1 (Central Valley) and site 7 (Cordillera de Los Andes), south-central Chile.

taminadas, donde los valores registran un significativo incremento en verano. En el período primavera-verano con el aumento de las temperaturas es posible constatar un incremento de la depositación de nitrógeno, pero las fuentes gaseosas pueden ser diferentes. En el sur de Chile no sólo la ganadería intensiva sería responsable de las emisiones nitrogenadas, sino también el incremento de procesos de denitrificación en el suelo saturado por las precipitaciones abundantes durante el invierno y que producen $\mathrm{N}_{2} \mathrm{O}$, aunque en el presente trabajo estos flujos no han sido estimados. Una comprobación más rigurosa del origen de los nutrientes requeriría conocer las masas de isotópicas de las formas de nitrógeno en los fertilizantes agrícolas o, productos de lechería, y aquellos encontrados en la lluvia.

Otro aspecto a considerar es el aporte de compuestos nitrogenados vía nubes o neblinas, las cuales se desplazan en forma horizontal e impactan en el dosel de los bosques situados en la alta montaña, fenómeno frecuente en el área de estudio. Valores de referencia de componentes químicos para el sitio 7 del transecto, que consideran mediciones comparativas del agua de neblina y de precipitación para 10 eventos durante 1999, indican un enriquecimiento en $\mathrm{N}$-inorgánico en la neblina, particularmente nitrato (promedio $=87,1$ $\mu \mathrm{g} \mathrm{L}^{-1}$ ), valor cinco veces mayor al agua de precipitación (promedio $=16,7 \mu \mathrm{g} \mathrm{L}^{-1}$ ) (datos no publicados). Esta fuente de ingreso ha sido evaluada en el Parque Nacional Torres del Paine (53 ${ }^{\circ}$ S) (Weathers \& Likens 1997), comprobando ingresos de nitrato y amonio, cuyos valores son comparativamente superiores a los registrados para Alaska y Oregon (Weathers et al. 1988). Se ha sugerido, que esta podría ser una fuente importante de ingreso en aquellos ecosistemas 
deficitarios en nitrógeno, como son los bosques del sur de Chile (Weathers \& Likens 1997). Los eventos de neblina y aportes de compuestos nitrogenados, constituyen a menudo ingresos ocultos necesarios de cuantificar en futuros estudios hidrológicos y biogeoquímicos, para ecosistemas boscosos de alta montaña (Weathers 1999).

Las tasas anuales de depositación atmosférica de $\mathrm{NO}_{3}-\mathrm{N}$ fluctuaron entre 0,53 y $0,57 \mathrm{~kg} \mathrm{ha}^{-1}$ año${ }^{1} \mathrm{y}$ de $\mathrm{NH}_{4}-\mathrm{N}$ fueron entre 6,4 y $2,8 \mathrm{~kg} \mathrm{ha}^{-1} \mathrm{año}^{-1}$, en las zonas agrícola-ganaderas del valle longitudinal (sitio 1) y el área boscosa de la cordillera de Los Andes (sitio 7), respectivamente (Fig. 4A). De acuerdo a los valores normalizados, considerando las diferencias de precipitación entre los sitios, se observa que las tasas de depositación de $\mathrm{NO}_{3}-\mathrm{N}$ en los sitios agrícolaganaderos fluctuaron $0,48-0,23 \mathrm{~g} \mathrm{ha}^{-1} \mathrm{~mm}^{-1} \mathrm{año}^{-1}$, y en los sitios de áreas boscosas entre $0,1-0,08 \mathrm{~g} \mathrm{ha}^{-}$ ${ }^{1} \mathrm{~mm}^{-1}$ año ${ }^{-1}$ (Fig. 4B). Respecto del $\mathrm{NH}_{4}-\mathrm{N}$ estos valores variaron entre 5,79 y $0,42 \mathrm{~g} \mathrm{ha}^{-1} \mathrm{~mm}^{-1}$ año-1 $^{-1}$ en los sitios 1 y 7 , respectivamente (Fig. 4B).

Se debe considerar que los valores de las tasas de depositación nitrogenada determinadas en este estudio, pueden subestimar el aporte total al suelo y vegetación, debido a que no se ha considerado la neblina. Antecedentes sobre estimaciones de depositación de nitrógeno por neblina para la zona de Antillanca (sitio 7) son de 0,9 y 1,4 kg $\mathrm{ha}^{-1}$ año ${ }^{-1}$, para $\mathrm{NO}_{3}-\mathrm{N}$ y $\mathrm{NH}_{4}-\mathrm{N}$, respectivamente (datos no publicados). Otra alternativa de ingreso es vía depositación seca, proceso durante el cual gases o material particulado son transportados desde la atmósfera y depositados sobre la superficie del suelo y/o vegetación, sin considerar la participación de la precipitación (Duyzer et al.

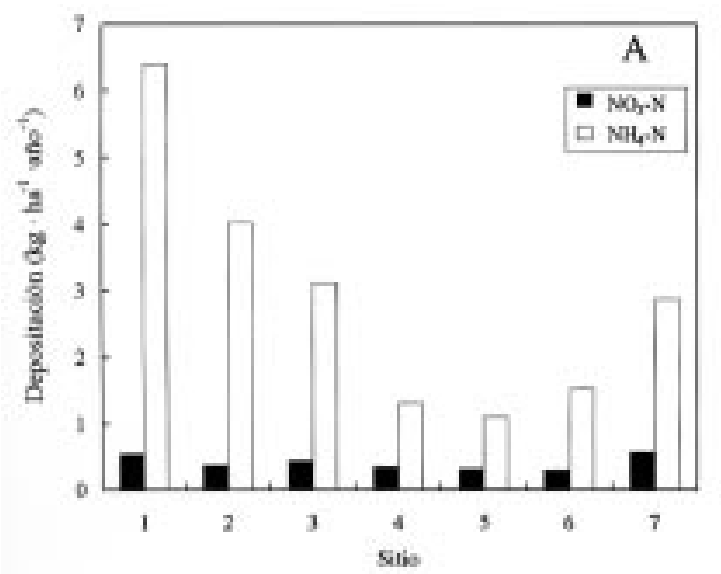

1992). Este aporte es de importancia en períodos de primavera-verano, cuando las precipitaciones son escasas, aumenta la temperatura y existe un notorio incremento de las actividades agrícola ganaderas en la región, con las consecuentes emisiones de $\mathrm{NH}_{3}$, producto de la aplicación de fertilizantes y purines. Esta última práctica, cada vez más frecuente en zonas con ganadería intensiva, produce liberación de amoniaco vía descomposición microbiana de la urea y desde muchas otras fracciones de nitrógeno de la orina del ganado (Fangmeier et al. 1994, Rennenberg \& Gessler 1999). Antecedentes del INE (1997) señalan para la provincia de Osorno un número superior a 510.000 vacunos, la cual constituye una masa ganadera importante a considerar como responsable de las emisiones de $\mathrm{NH}_{3}$. Un monitoreo preliminar de depositación seca de $\mathrm{NH}_{3}$ con filtros SAM, realizado en la región ganadera del Valle Central cerca de Paillaco $\left(40^{\circ} 07^{\prime} \mathrm{S}\right)$, durante el período noviembre 2000-enero 2001, entregan valores cercanos a 2,5 kg ha ${ }^{-1} \mathrm{mes}^{-1}$ (datos no publicados). Montos de depositación de compuestos nitrogenados reducidos $\left(\mathrm{NH}_{\mathrm{y}}\right)$ en Alemania, señalan contribuciones proporcionalmente mayores vía depositación seca versus depositación húmeda, con 9,4 y 7,0 $\mathrm{kg} \mathrm{ha}^{-1} \mathrm{año}^{-1}$, respectivamente (Fangmeier et al. 1994). La importancia de la depositación seca, también ha sido indicada en mediciones en Chile central $\left(33^{\circ} \mathrm{S}\right)$, donde las contribuciones de compuestos nitrogenados $\left(\mathrm{NO}_{3}{ }^{-}\right.$ y $\mathrm{NH}_{4}^{+}$) mediante esta vía, fueron comparativamente mayores que la depositación húmeda (Prado-Fiedler \& Fuenzalida 1996).

Las altas depositaciones de $\mathrm{NH}_{4}^{+}$tienen un efecto en la química de la solución del suelo, intensifi-

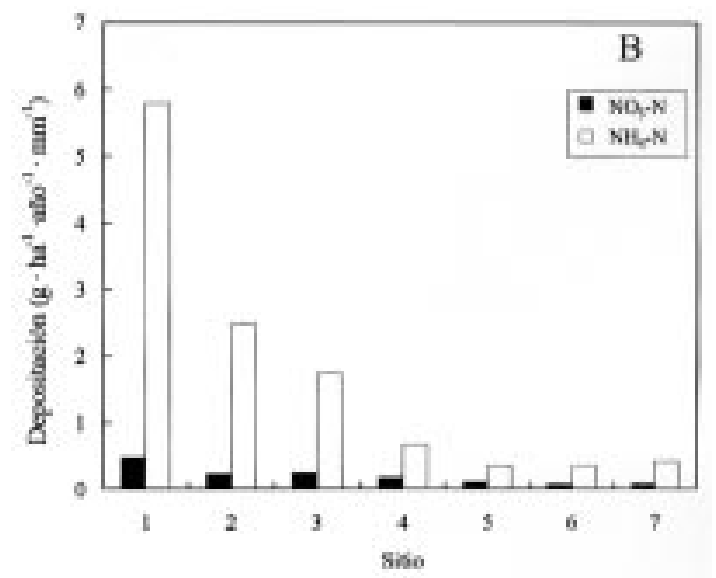

Fig. 4: Depositación atmosférica de nitrógeno inorgánico total (A) y normalizado (B) del transecto desde los sitios agrícola-ganaderos en el valle longitudinal hacia los bosques de la cordillera de Los Andes, centro-sur de Chile.

Atmospheric deposition of total (A) and normalized inorganic-N (B) along a transect from agricultural-livestock sites of the central valley to forests in the Cordillera de Los Andes, south-central Chile. 
cando la nitrificación y la acidificación, lavado de $\mathrm{NO}_{3}^{-}$y cationes, y movilización de aluminio con su consecuente daño a las raíces (Carnol et al. 1997). Este desbalance de nutrientes en los ecosistemas terrestres, conduce a un incremento de la relación $\mathrm{NH}_{4}^{+}$/cationes en el suelo, lo que ha sido mencionado como una de las causas del actual daño en los bosques de Europa (Schulze 2000). Las deficiencias de fósforo y potasio en las plantas inhiben la captación de nitrógeno por las raíces, produciendo una mayor pérdida por efecto de lavado e incorporación de nitrógeno inorgánico a las napas freáticas (Rennenberg \& Gessler 1999). Por otro lado, se ha sugerido que la retención de nitrógeno inorgánico en el suelo es de carácter diferencial, existiendo una mayor retención de nitrógeno en forma de amonio, cuando estas formas son superiores a las de nitrato (Aber et al. 1998).

En el período pre-industrial, el nitrógeno era el mayor factor limitante en los ecosistemas terrestres no agrícolas (Galloway 1995). En consecuencia, los procesos microbiológicos en el suelo del bosque, metabólicos de las plantas y funcionamiento en general del ecosistema, estarían más adaptados a la condición de limitación de nitrógeno que a un exceso del mismo (Rennenberg \& Gessler 1999). Por lo que frente a un futuro escenario de incremento en la depositación de nitrógeno vía atmósfera, constituye un riesgo potencial para la vegetación nativa, al provocar un desbalance de nutrientes y favoreciendo aquellas especies de carácter nitrófila, situación mencionada como una de las causas del actual daño en las regiones con altas tasas de depositación de nitrógeno (Schulze 2000).

Es importante destacar el potencial efecto de la depositación atmosférica de nitrógeno y la aplicación de fertilizantes al suelo, las cuales pueden influir la composición química del agua de percolación que alcanza las napas freáticas. Nissen et al. (2000) ha documentado que en las aguas subterráneas del valle longitudinal $\left(41^{\circ} \mathrm{S}\right)$, las concentraciones de amonio, manganeso, fosfato y aluminio, presentan valores muy superiores a los límites permitidos como fuente de agua potable, incluso algunos con contaminación microbiológica. Antecedentes de Rojas (1990), indican un incremento en el uso de fertilizantes en Chile, con una mayor demanda de compuestos nitrogenados en la producción de forraje para lechería y ganadería. Datos de estudios realizados en microcuencas con bosques nativos y agrícola-ganaderas cercanas al lago Rupanco (Oyarzún et al. 1997), indican aumentos significativos en las salidas de nitrato vía escorrentía superficial en las cuencas con actividades agrícolas, especialmente praderas ganaderas. Esto ha sido atribuido al incremento de las actividades forestales y a la intensificación de la utilización de fertilizantes en las actividades agrícolas.

\section{CONCLUSIONES}

Los resultados entregan evidencias de depositación atmosférica de compuestos nitrogenados reducidos, los que estarían siendo originados por las emisiones de nutrientes de la creciente actividad agrícola y ganadera desarrollada en el valle longitudinal del centro-sur de Chile, ya que principalmente el $\mathrm{NH}_{4}+$ es depositado cercano a las fuentes de emisión vía depositación húmeda y seca. Aunque las concentraciones de $\mathrm{NO}_{3}-\mathrm{N}$ en el agua de precipitación en las áreas agrícolas y ganaderas son significativamente mayores que en áreas boscosas, los valores reflejan una escasa influencia de las actividades industriales en la región, al compararlas con áreas altamente impactadas por actividades antropogénicas en el hemisferio norte. En cambio, las altas concentraciones de $\mathrm{NH}_{4}-\mathrm{N}$ evidencian el impacto de las actividades ganaderas, especialmente en el valle longitudinal.

Las tasas de depositación de nitrógeno inorgánico vía precipitación húmeda en el valle longitudinal, cerca de la ciudad de Osorno, son de $7,1 \mathrm{~kg} \mathrm{ha}^{-1} \mathrm{año}^{-1}$. Sin embargo, este valor estaría subestimando los aportes totales al no considerar la contribución de la depositación seca en períodos primavera-verano, los cuales podrían hasta duplicar los aportes totales de nitrógeno al suelo y vegetación, aspecto necesario a considerar en estudios posteriores. Mientras que las tasas de depositación en los bosques de la cordillera de Los Andes $\left(3,4 \mathrm{~kg} \mathrm{ha}^{-1}\right.$ año $\left.{ }^{-1}\right)$, también estarían subestimadas al no ser considerados los aportes vía nubes y neblinas, especialmente en los bosques situados a mayor altitud.

La creciente depositación atmosférica de compuestos nitrogenados a escala regional y global, sumado al aumento de las actividades agrícolas y ganaderas con la aplicación de purines, podría influir significativamente la química del suelo, agua de escorrentía superficial y napas freáticas, provocando un desbalance de nutrientes en los ecosistemas regionales.

\section{AGRADECIMIENTOS}

Este estudio ha sido financiado por el proyecto No. 1990694 del Fondo Nacional de Ciencias y Tecnología, proyecto No. 2000-07 de la Direc- 
ción de Investigación y Desarrollo de la Universidad Austral de Chile, y por el Proyecto Bilateral Chile-Flanders BIL 99-4. Se agradece la colaboración de los propietarios que colaboraron con su disposición para acceder a los sitios del transecto de estudio, al Club Andino Antillanca, Corporación Nacional Forestal X Región y en especial, la colaboración del guardaparques Sr. Nicolás Pacheco por su ayuda en labores de terreno.

\section{LITERATURA CITADA}

ABER JD, MC DOWELL, KJ NADELHOFER, K MAGILL, A BERNSTON, G KARNAKEA, M MCNULTY, S CURRIE, W RUSTAD \& Y FERNÁNDEZ (1998) Nitrogen saturation in temperate forest ecosystems. BioScience 48: 921-934.

ANRIQUE R, L LATRILLE, O BALOCCHI, D ALOMAR, V MOREIRA, R SMITH, D PINOCHET \& G VARGAS (1999) Competitividad de la producción lechera nacional. Resumen Ejecutivo, Universidad Austral de Chile, Facultad de Ciencias Agrarias, Volumen 1. 216 pp.

APHA-AWWA-WEF (1995) Standard methods for the examination of water and wastewater. Decimonovena edición. American Public Health Association, Washington, Distrito de Columbia. 157 pp.

BLEW R \& R EDMONDS (1995) Precipitation chemistry along an inland transect on the Olympic Peninsula, Washington. Journal of Environmental Quality 24: 239-245.

CARNOL M, P INESON, JM ANDERSON, F BEESE, MP BERG, T BOLGER, M-M COUTEAUX, P CUDLIN, S DOLAN, M RAUBUCH \& HA VERHOEF (1997) The effects of ammonium sulphate deposition and root sinks on soil solution chemistry in coniferous forest soils. Biogeochemistry 38: 255-280.

DE SCHRIJVER A, G VAN HOYDONCK, L NACHTERGALE, L DE KEERSMAEKER, S MUSCHE \& N LUST (2000) Comparison of nitrate leaching under silver beech (Betula pendula) and corsican pine (Pinus nigra spp. Lauricio) in Flanders (Belgium). Water, Air \& Soil Pollution 122: 77-91.

DISE NB, ED MATZNER \& P GUNDERSEN (1998) Synthesis of nitrogen pools and fluxes from european forest ecosystems. Water, Air \& Soil Pollution 105: 143-154.

DUYSER JH, HL VERHAGEN, JH WESTRATE \& FC BOSVELD (1992) Measurement of dry deposition flux of $\mathrm{NH}_{3}$ onto coniferous forest. Environmental Pollution 75: 3-13.

FANGMEIER A, A HADWIGER-FANGMEIER, L VAN DER EERDEN \& H-J JÄEGER (1994) Effects of atmospheric ammonia on vegetation-a review. Environmental Pollution 86: 43-82.

GALLOWAY JN (1995) Acid deposition: Perspectives in time ad space. Water, Air \& Soil Pollution 85: 15-24.
GALLOWAY JN, WC KEENE \& GE LIKENS (1996) Processes controlling the composition of precipitation at a remote southern hemisphere location: Torres del Paine National Park, Chile. Journal of Geophysical Research 101: 6883-6987.

GODOY R, C OYARZÚN \& J BAHAMONDES (1999) Flujos hidroquímicos en un bosque de Nothofagus pumilio en el Parque Nacional Puyehue, sur de Chile. Revista Chilena de Historia Natural 72: 579-594.

GODOY R, C OYARZÚN \& V GERDING (2001) Precipitation chemistry in deciduous and evergreen Nothofagus forests of southern Chile under a lowdeposition climate. Basic and Applied Ecology 2: 65-72.

HEDIN LO \& H CAMPOS (1991) Importance of small streams in understanding and comparing watershed ecosystem processes. Revista Chilena de Historia Natural 64: 583-596.

HEDIN LO \& ED HETHERINGTON (1996) Atmospheric and geologic constrains on the biogeochemistry of North and South American temperate rainforest. En: Lawford R, P Alaback \& E Fuentes (eds) High-altitude rainforest and associated ecosystems of the west coast of rthe Americas: climate, hydrology, ecology and conservation: 57-74. Springer Verlag, New York, New York.

HEDIN LO, J ARMESTO \& AH JOHNSON (1995) Patterns of nutrient loss from unpolluted, old growth temperate forest: evaluation of biogeochemical theory. Ecology 76: 493-509.

INSTITUTO NACIONAL DE ESTADÍSTICA, INE (1997) Sexto censo nacional agropecuario: resultados preliminares. Impresos Universitaria, Santiago, Chile. 443 pp.

KLEMOLA S \& G SOEDERMAN (1993) Manual for integrated monitoring. International Co-operative Programme on Integral Monitoring on Air Pollution Effects. Environmental Report 5. Environment Data Centre, National Board of Water and the Environment, Helsinski, Finlania. 114 pp.

LIKENS GE \& FH BORMANN (1995) Biogeochemistry of a forested ecosystems. Springer-Verlag, New York, New York. 159 pp.

LIKENS GE, WC KEENE, JM MILLER \& D GALLOWAY (1987) Chemistry of precipitation from remote, terrestrial site in Australia. Journal of Geophysical Research 92: 13299-13314.

NISSEN J M, M GARAY, A AGUILERA \& E VALENZUELA (2000) Calidad de aguas subterráneas en la décima región de Chile. Agro Sur (Chile) 28: $25-39$.

OYARZÚN C E, H CAMPOS \& A HUBER (1997) Exportación de nutrientes en microcuencas con distinto uso del suelo en el sur de Chile (Lago Rupanco, X Región). Revista Chilena de Historia Natural 70: 507-519.

OYARZÚN CE, R GODOY \& A SEPÚLVEDA (1998) Water and nutrient fluxes in a cool temperate rainforest at the Cordillera de la Costa in southern Chile. Hydrological Processes 12: 1067-1077.

PÉREZ C, L HEDIN \& J ARMESTO (1998) Nitrogen mineralization in two unpolluted old growth forest of contrasting biodiversity and dynamics. Ecosystems 1: 361-373. 
PRADO-FIEDLER R \& H FUENZALIDA (1996) Wet and dry deposition of nitrogen compounds in the southeast Pacific coast: Montemar, central Chile. Journal of Geophysical Research 101: 22845-22853.

RENNENBERG H \& A GESSLER (1999) Consequences of $\mathrm{N}$ depositation to forest ecosystems-recent results and future research. Water, Air \& Soil Pollution 116: 47-64.

REYNOLS B, E WILSON \& B EMMETT (1998) Evaluating critical loads of nutrient nitrogen and acidity for terrestrial systems using ecosystem-scale experiments (NITREX). Forest Ecology and Management 101: 8194.

ROJAS C (1990) Análisis del consumo de fertilizantes en Chile y el mundo. Informativo Agroeconómico Fundación Chile, agosto: 51-57.

SCHULZ EF (1989) Problems in applied hydrology. Water Resources Publications, Littleton, Colorado. 489 pp.

SCHULZE E-D (2000) The carbon and nitrogen cycle of forest ecosystems. En: Schulze E-D (ed) Carbon and nitrogen cycling in European forest ecosystem: 3-11. Springer-Verlag, Berlin, Alemania.
SOKAL RR \& FJ ROHL (1981) Biometry: the principles and practices of statistics in biological research. W.H. Freeman, San Francisco, California.

SUTTON M, DS LEE, G DOLLARD \& D FOELER (1998) Atmospheric ammonia: emission, deposition and environmental impacts. Atmospheric Environment 32: 269-271.

TORSETH K \& A SEMB (1997) Atmospheric deposition of nitrogen, sulphur and chloride in two watersheds located in southern Norway. Ambio 26: 258-265.

WEATHERS K (1999) The importance of cloud and fog in the maintenance of ecosystems. Trends in Ecology and Evolution 14: 214-215.

WEATHERS K \& G LIKENS (1997) Clouds in southern Chile: an important source of nitrogen to nitrogenlimited ecosystems? Environmental Science \& Technology 31: 210-213.

WEATHERS K, G LIKENS, F BORMAN, S BICKNELL, H BORMAN, B DAUBE, J EATON, J GALLOWAY, W KEENE, K KIMBALL, W McDOWELL, T SICCAMA, D SMILEY \& R TARRANT (1988) Cloud water chemistry from ten sites in North America. Environmental Science \& Technology 22: 1018-1026.

Editor Asociado: J. Armesto

Recibido el 30 de marzo de 2001; aceptado el 3 de diciembre de 2001 